\title{
The role of basal-plane oxygen atoms in determining the ferroelastic and microstructural properties of $\mathrm{Y}-\mathrm{Ba}-\mathrm{Cu}-\mathrm{O}$
}

\author{
V K WADHAWAN* \\ Neutron Physics Division, Bhabha Atomic Research Centre. Trombay, Bombay 400085 , \\ Indore 452 013, India \\ *Present address: Materials Group, Laser Programme, Centre for Advanced Technology, \\ Indore 452013, India
}

\begin{abstract}
The basal-plane oxygen atoms in $\mathrm{YBa}_{2} \mathrm{Cu}_{3} \mathrm{O}_{7-x}$ behave like a lattice gas, with very high diffusivity, especially for oxygen-deficient specimens. Implicit in this behaviour is the property that even a small amount of stress applied along the $b$-axis $(b>a)$ can make these oxygen atoms hop from the $\left(0, \frac{1}{2}, 0\right)$ sites to the $\left(\frac{1}{2}, 0,0\right)$ sites. This is suggested as the primary mechanism responsible for the ferroelastic switching observed in this crystal. Since the material is an oxide of a mixed-valence element $(\mathrm{Cu})$, the common occurrence of overall nonstoichiometry is only to be expected. Also, as discussed by Khachaturyan and others, except at very high temperature, the oxygen atoms and the vacancies will always have a tendency for ordering and/or precipitation into configurations which approach near-perfect stoichiometry locally. However, not all evidence for ordering is in conformity with the predictions of Khachaturyan's concentration-wave model. The experimental data are examined critically. Further experiments to resolve the discrepancies are suggested.
\end{abstract}

Keywords. Ferroelastics; $\mathrm{Y}-\mathrm{Ba}-\mathrm{Cu}-\mathrm{O}$; phase transitions; ordering; precipitation: disorientations.

\section{Introduction}

The basal-plane oxygen atoms in $\mathrm{YBa}_{2} \mathrm{Cu}_{3} \mathrm{O}_{7-x}(\mathrm{Y}-\mathrm{Ba}-\mathrm{Cu}-\mathrm{O})$ behave like a lattice gas (Varea and Robledo 1988; Bartelt et al 1989), with a very high diffusivity (Cannelli et al 1988). In the high-temperature tetragonal phase the $\left(0, \frac{1}{2}, 0\right)$ and $\left(\frac{1}{2}, 0,0\right)$ basal-plane sites are equally occupied by oxygen atoms. On cooling the material a high-temperature phase transition to the orthorhombic phase occurs. In the orthorhombic phase the $\left(0, \frac{1}{2}, 0\right)$ occupancy is higher than that of $\left(\frac{1}{2}, 0,0\right)$. Since these two sites are symmetry-equivalent in the tetragonal phase, either of them can become the $\left(0, \frac{1}{2}, 0\right)$ site in the orthorhombic phase, with the other becoming the $\left(\frac{1}{2}, 0,0\right)$ site. This is the primary reason for the occurrence of the ubiquitous $\{110\}$ twin walls in this material. In the jargon of the theory of ferroelasticity, the two twin components separated by these walls are referred to as two distinct orientation states (Wadhawan $1982,1988)$. Because of the preferential occupation of the $\left(0, \frac{1}{2}, 0\right)$ sites by oxygen atoms, the lattice parameter $b$ is slightly greater than the parameter $a$. If a uniaxial compressive stress is applied along the $b$-axis of a single crystal of $\mathrm{Y}-\mathrm{Ba}-\mathrm{Cu}-\mathrm{O}$, then, if the stress exceeds the coercive stress for that temperature, the crystal undergoes a state shift; that is, the $a$ - and $b$-axes interchange their identities, so that the smaller axis (a-axis) is now aligned along the direction of compression (Somayazulu et al 1989). This is referred to as the ferroelastic effect (Wadhawan 1982). At the atomic level this effect is brought about primarily by the ease with which the basal-plane oxygens can hop from the $\left(0, \frac{1}{2}, 0\right)$ position to the $\left(\frac{1}{2}, 0,0\right)$ position under the influence of the applied mechanical bias. This ferroelastic switchability has a number of practical implications, which are described briefly in $\$ 2$. 
Another important feature of $\mathrm{Y}-\mathrm{Ba}-\mathrm{Cu}-\mathrm{O}$ is the strong tendency for the basal-plane oxygens to precipitate and/or order into a variety of configurations. The reasons for this are thermodynamic as well as chemical (because of the mixed valence that $\mathrm{Cu}$ can exhibit). In $\S 3$ we describe very briefly the two main contending models for oxygen or vacancy ordering. The models do not lead to the same predictions. Some of the experimental evidence for vacancy ordering is critically reviewed. In $\S$ 4 it is proposed that some of the apparent discrepancies can be explained by taking due note of the occurrence of ferroelastic disorientations (Wadhawan 1988).

\section{Some applications of the ferroelastic nature of $\mathrm{Y}-\mathrm{Ba}-\mathrm{Cu}-\mathrm{O}$}

The ferroelastic nature of $\mathrm{Y}-\mathrm{Ba}-\mathrm{Cu}-\mathrm{O}$ implies that the transformation-twin walls occurring in it can be moved, and therefore removed, by the application of an appropriate uniaxial stress. This has indeed been demonstrated by Somayazulu et al (1989), who could achieve detwinning even at room temperature. The influence of twin walls on the superconducting properties of $\mathrm{Y}-\mathrm{Ba}-\mathrm{Cu}-\mathrm{O}$ is still a matter of active investigation. Some workers have argued in support of twinning-plane superconductivity in this material (Abrikosov et al 1988). Others believe that the presence of these walls has a deleterious effect on the critical current density (Deutscher and Muller 1987). There is also some evidence for pinning of the magnetic flux lines at twin planes at temperatures near the diamagnetic transition temperature (Liu et al 1989). Although the true picture regarding the influence of twin planes on the superconducting properties is far from clear, it is, nevertheless, important that the ferroelastic nature of the material allows the domain structure to be manipulated by the application of a suitable external stress.

Another possible application of the ferroelastic property of $\mathrm{Y}-\mathrm{Ba}-\mathrm{Cu}-\mathrm{O}$ involves the conversion of a single crystal to a bicrystal by a simple bending process (Wadhawan 1989). Such a bicrystal can act as an S-N-S Josephson-tunnelling device. In the proposed procedure, a crystal of the material is cut into the shape of a rectangular bar with edges parallel to the three crystallographic axes (figure 1a). The dimensions are to be so chosen that the $b$-axis is along the length of the bar, and the thinnest dimension is along the $c$-axis. Such a configuration should be easy to

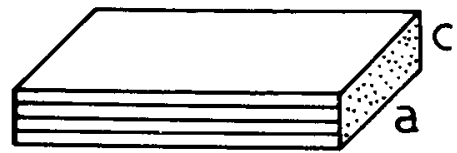

b

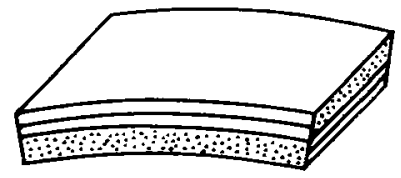

(a)

(b)

Figure 1. A proposed configuration for converting a single crystal of $\mathrm{Y}-\mathrm{Ba}-\mathrm{Cu}-\mathrm{O}$ to a bicrystal. (a) Single crystal before bending. (b) Bicrystal resulting from the bending of the single crystal in the $b c$-plane. Note the interchange of the $a$ - and the $b$-axes in the lower half of the bicrystal. 
produce because the $a b$-plane is the cleavage plane, as well as a growth-habit plane. Suppose this bar is subjected to bending in the $b c$-plane, layers of the crystal above a certain 'neutral' plane will be subjected to elongation, while those below it will be compressed along the $b$-direction. Since $a<b$, the compressed layers will undergo ferroelastic switching as so to present the shorter axis ( $a$-axis) along the direction of compression. As shown in figure $1 \mathrm{~b}$, the result will be a bicrystal, with a (presumably non-superconducting) twist-boundary separating two monocrystals rotated by $90^{\circ}$ with respect to each other in the $a b$-plane. In fact, in an actual device application, a variety of bending configurations can be produced and maintained by simply turning some appropriately positioned bending screws.

The third practical application of the ferroelastic property of $\mathrm{Y}-\mathrm{Ba}-\mathrm{Cu}-\mathrm{O}$ can be for producing three-dimensional alignment of grains of the ceramic or the composite (Wadhawan 1989). Such an alignment is expected to result in an order-of-magnitude increase in the critical current density (Farrel et al 1987; Dimos et al 1988). The proposed procedure is an extension of the method followed by Farrell et al (1987), who could achieve two-dimensional alignment of grains by applying a strong (9.4 T) magnetic field to a mixture of small crystals of $\mathrm{Y}-\mathrm{Ba}-\mathrm{Cu}-\mathrm{O}$ and an epoxy resin during the final stages of the setting of the epoxy. If a compressive mechanical stress is also applied in a direction perpendicular to the magnetic field, ferroelastic reorientation of the twin domains would occur so as to make the $a$-axis preferentially point along the direction of compression. If it also becomes possible to get rid of the epoxy resin (or some other binder material), and to make the grains fuse together without losing their alignment, we would have produced a large pseudocrystal of $\mathrm{Y}-\mathrm{Ba}-\mathrm{Cu}-\mathrm{O}$.

\section{Vacancy ordering in $\mathrm{Y}-\mathrm{Ba}-\mathrm{Cu}-\mathrm{O}$}

Non-stoichiometric oxides of a mixed-valence element like $\mathrm{Cu}$ can exist in a unique-structure single-phase configuration only at very high temperatures (Anderson 1973; Khachaturyan 1983). As the temperature is lowered, the configurational entropy must decrease (and eventually become zero at $T=0$, as required by the third law of thermodynamics). Ordering and/or precipitation of oxygen atoms and vacancies can therefore be expected for $\mathrm{Y}-\mathrm{Ba}-\mathrm{Cu}-\mathrm{O}$ at lower temperatures (Wadhawan and Bhagwat 1989). The exact form of the resultant phase diagram depends on the nature of the short-range and long-range interactions. According to the concentration-wave model of Khachaturyan and Morris (1988), in addition to the decomposition into tetragonal and orthorhombic phases at appropriate temperatures and concentrations, a homologous series of transient ordered structures having the stoichiometry $\mathrm{YBa}_{2} \mathrm{Cu}_{3} \mathrm{O}_{7-n /(2 n+1)}(n=0,1,2, \ldots)$ may form during the spinodal decomposition of the material.

The predictions of the above model are at variance with the model proposed by Alario-Franco et al (1988), who observed (by electron diffraction) ordered structures which are well-described by the oxygen stoichiometry $7-n / 8$, rather than $7-n /(2 n+1)$. Crystal-chemical arguments explaining the observations of AlarioFranco et al (1988) have been given by Goodenough and Manthiram (1988). In view of this controversy, we examine here the experimental evidence cited by Khachaturyan and Morris (1988) in support of their model. According to this model, diffraction 
peaks are expected at $\frac{1}{3}, \frac{2}{5}, \frac{3}{7}, \ldots, \frac{1}{2}$ along the $a^{*}$ direction. The diffraction peak at $\left(\frac{1}{2}, 0,0\right)$ is, in fact, predicted by both the models, and has been reported by many workers. It corresponds to a periodicity of $2 a_{0}$ in real space, with $a_{0} \approx 3.8 \AA$.

Van Tendeloo et al (1987) observed by HREM a lattice periodicity of $3 \mathbf{a}_{0}$, occurring locally in patches. However, these intensity modulations in the HREM picture were too weak to manifest themselves as superlattice reflections at $\left(\frac{1}{3}\right) \mathrm{a}^{*}$. The evidence does not appear to be of a conclusive nature.

Werder et al (1988) published selected-area electron diffraction patterns exhibiting narrow diffuse streaking which, according to the authors, are centred around $\left(\frac{2}{5}\right) a^{*}$. It should be noted, however, $\frac{2}{5}$ differs from $\frac{3}{8}$ (the fraction resulting from the model of Alario-Franco et al (1988)) by only $\frac{1}{40^{\circ}}$. This may well be within the experimental error. In real space, if we compute the corresponding oxygen stoichiometry, then $7-\frac{2}{5}=6.6$ and $7-\frac{3}{8}=6.625$. The difference is not easy to detect experimentally.

We next consider the work of You et al (1988) who carried out high-resolution synchrotron XRD studies on (twinned) single crystals of the material. They concluded from their data on a specimen with $\{110\}$ twins that the specimen has subgrains, each of which has a value of the orthorhombicity parameter $(b-a) / a$ differing by as little as 0.003 from the others. This work cannot distinguish between the models of Khachaturyan and Morris (1988) and Alario-Franco et al (1988). Moreover, some of the data can be given a different interpretation in terms of the occurrence of ferroelastic disorientations. The basic idea (discussed in detail by Wadhawan 1988) is that, because of the ferroelastic nature of the material, and because of the fact that the basal-plane oxygens have a high diffusivity, a large number of closely-spaced orientations of domains can occur in many of the grains. It is not at all easy to unscramble the effect of these disorientations and the effect of genuine variations in $(b-a) / a$ on the final XRD pattern.

\section{Discussions and conclusions}

The multiphase character of $\mathrm{Y}-\mathrm{Ba}-\mathrm{Cu}-\mathrm{O}$ at the usual temperatures of interest is well established, as expected. But there is no consensus yet on the exact nature of the vacancy ordering. There exists evidence for a variety of structures, not all of which appear to be mutually compatible. More experiments on carefully characterized samples are called for. It appears to the present author that gamma-ray diffractometry can help resolve some of the discrepancies (Wadhawan 1988). This is because, being a forward-scattering experiment, it can readily distinguish between the contributions from stoichiometry variations and those from the disorientations mentioned above.

\section{References}

Abrikosov A A, Buzdin A I, Kulic M L and Kuptsov D A 1988 Int. J. Mod. Phys. 11045

Alario-Franco M A, Chaillout C, Capponi J J, Chenavas J and Marezio M 1988 Physica C156 455

Anderson J S $1973 \mathrm{~J}$. Chem. Soc. Dalton Trans. 1107

Bartelt N C, Einstein T L and Wille L T 1989 Phys. Rev. B40 10759

Cannelli G, Cantelli R and Cordero F 1988 Phys. Rev. B38 7200

Deutscher G and Mueller K A 1987 Phys. Rev. B37 5837

Dimos D, Choudhury P, Mannhart J and LeGoues F K 1988 Phys. Rev. Lett. 61219 
Farrell D E et al 1987 Phys. Rev. B36 4025

Goodenough J B and Manthiram A 1988 in High temperature superconductors (eds) J Heiras et al (Singapore: World Scientific) pp. 18-27

Khachaturyan A G 1983 Theory of structural transformations in solids (New York: Wiley)

Khachaturyan A G, and Morris J W 1988 Phys. Rev. Lett. 61215

Liu J Z, Lan M D, Klavins P and Shelton R N 1989 (Preprint)

Somayazulu M S, Rao S M D and Wadhawan V K 1989 Mater. Res. Bull. 24795

Van Tendeloo G, Zandbergen H W and Amelinckx S 1987 Solid State Commun. 63603

Varea C and Robledo A 1988 in High temperature superconductors (eds) J Heiras et al (Singapore: World Scientific) pp. 145-153

Wadhawan V K 1982 Phase Transitions 33

Wadhawan V K 1988 Phys. Rev. B38 8936

Wadhawan V K 1989 Ferroelectrics 97171

Wadhawan V K and Bhagwat K V 1989 Phase Transitions 1927

Werder D J, Chen C H, Cava R J and Batlogg B 1988 Phys. Rev. B37 2317

You H, Axe J D, Kan X B, Moss S C, Liu J Z and Lam D J 1988 Phys. Rev. B37 2301 